\title{
Array calibration method for gain-phase errors based on asynchronous interstation direct wave interference
}

\author{
Liu Bin ${ }^{\text {a) }}$, Wu Xiongbin ${ }^{\text {b) }}$, Li Lun, Xu Xing'an, and Long Chao \\ Electronic Information Institute of Wuhan University, Wuhan, China, 430072 \\ a)liubinkingmax@yahoo.com.cn \\ b)xbwu@whu.edu.cn
}

\begin{abstract}
In this paper, we analyze the interstation direct wave interference (IDWI) under an asynchronous condition. We propose an array calibration method for the consistency of gain-phase for the first time and show the conditions and probability of the generation of IDWI and the characteristics of asynchronous IDWI. This method can implement calibration of direct wave without keeping radar stations in synchronous condition and perform outstanding precision and stability. The experimental data demonstrate that using array calibration for gain-phase errors based on asynchronous IDWI can get accurate and stable calibration values of gain-phase errors.
\end{abstract}

Keywords: asynchronism, interstation direct wave interference, FMICW, gain-phase errors, array calibration

Classification: Electron devices, circuits, and systems

\section{References}

[1] R. Schmidt, "Multiple emitter location and signal parameter estimation," IEEE Trans. Antennas Propag., vol. 34, no. 3, pp. 276-280, March 1986.

[2] M. Zhang and Z.D. Zhu, "DOA estimation with sensor gain, phase and position perturbations," Aerospace and Electronics Conference, vol. 1, pp. 67-69, March 1993.

[3] C.M.S. See, "Method for array calibration in high-resolution sensor array processing," Radar, Sonar and Navigation, IEEE Proceedings, vol. 142, no. 3, pp. 90-96, June 1995.

[4] B. Friedlander and A.J. Weiss, "Eigenstructure methods for direction finding with sensor gain and phase uncertainties," Acoustics, Speech, and Signal Processing, vol. 5, pp. 2681-2684, 1988.

[5] B. Friedlander and A.J. Weiss, "Direction finding in the presence of mutual coupling," IEEE Trans. Antennas Propag., vol. 39, no. 3, pp. 273-284, March 1991.

[6] X. Wu, F. Cheng, Z. Yang, and H. Ke, "Broad Beam HFSWR Array Calibration Using Sea Echoes," International Conference on Radar, pp. 13, Oct. 2006. 


\section{Introduction}

Array gain-phase errors can degrade the performance of super resolution algorithms such as MUSIC, and even make the algorithms fail. Hence, array calibration for gain-phase errors is necessary [1]. The method of array calibration for errors is mainly divided into two types, active calibration and passive calibration. The active calibration method estimates the array perturbation parameters by setting auxiliary source of which the direction is precisely known $[2,3]$; the passive calibration method usually makes joint estimation of direction of spatial signal source and array perturbation parameters, and this method can accomplish direction estimation and calibration in the same time without knowing the direction of auxiliary source $[4,5]$.

Antenna array of high-frequency surface wave radar (HFSWR) is close to the sea and it's expensive and inconvenient to use auxiliary source to calibrate, so passive calibration method is encouraged to develop. The passive calibration makes joint estimation of direction of spatial signal source and array element errors, but this method has high computation complexity and fuzzy estimation of parameters. A channel calibration technique which uses single DOA spectrum in the sea echo has been reported in the literature [6]. This technique uses statistical methods to deal with a large number of echo signals, and there is no complicated iterative operation. With low computation, this technique improves the real-time performance, accuracy and stability of channel calibration. But comparing with the active calibration method, the stability of this technique's calibration results is weaker.

In this paper, we propose an array calibration method for gain-phase errors based on asynchronous IDWI. This method can implement active calibration without auxiliary source and perform outstanding precision and stability. This method avoids iteration procedure of joint estimation of target and error parameters in the traditional self-calibration method and largely reduces the computation and time consuming.

\section{Analysis of IDWI}

\subsection{Signal model of IDWI}

Under ideal condition, Station A and Station B has a HFSWR respectively, and the distance between Station A and Station B is $R$. A frequency modulated interrupted continuous wave (FMICW) is employed as transmit waveform (see Fig. 1). The local frequencies of receivers in Station A and Station $\mathrm{B}$ are

$$
\begin{aligned}
& f_{A}(t)= \begin{cases}f_{A 0}-\alpha_{A}\left[t+\tau_{0}-(k-1) T\right] & -\tau_{0} \leq t-k T \leq T_{s}-\tau_{0} \\
0 & T_{s}-\tau_{0}<t-k T<T-\tau_{0}\end{cases} \\
& f_{B}(t)= \begin{cases}f_{B 0}-\alpha_{B}[t-(k-1) T] & 0 \leq t-k T \leq T_{s} \\
0 & T_{s}<t-k_{B} T<T\end{cases}
\end{aligned}
$$

where $\alpha_{A}=B_{A} / T_{s}, \alpha_{B}=B_{B} / T_{s}$, and $k=1,2,3, \cdots$ is the frame number.

After being mixed, the IDWI received by Station B has an output frequency as follow: 


$$
f_{B c p}(t)=m f_{A}\left(t-\tau_{Z D}\right)-n f_{B}(t) \pm l f_{q}
$$

where $m, n, l \in Z^{+}, f_{q}=1 / q, p=m+n+l$, and $p$ is the order of mixer's compound frequency component. The larger value of $p$ means the larger loss of IDWI. To get a large interference-to-noise ratio (INR) of frequency component of IDWI at baseband and make the frequency component a constant, $m \alpha_{A}=n \alpha_{B}, p \leq S$ (the value of $S$ is determined by radar system and interstation distance) is essential.

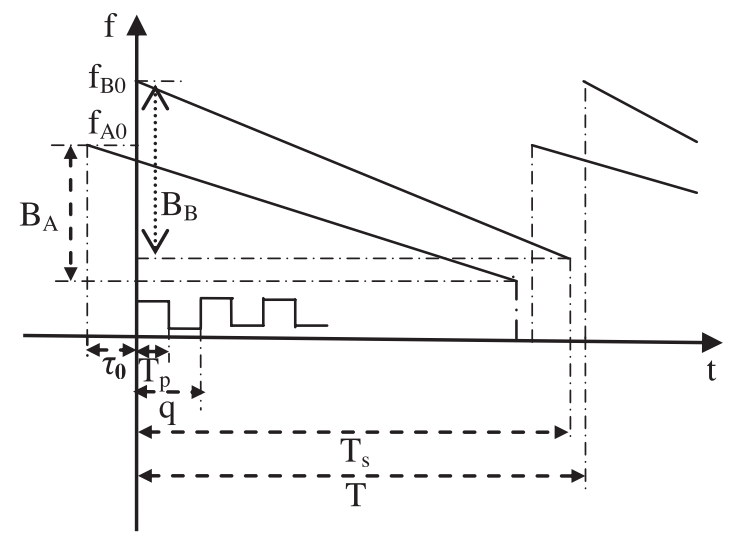

Fig. 1. Parameters of FMICW waveform. $f_{A 0}$ and $f_{B 0}$ are initial frequencies of Station A and Station B, respectively. $B_{A}$ and $B_{B}$ are sweep bandwidths of Station A and Station B, respectively. Radar at Station A starts earlier than radar at Station B and the time lag is $\tau_{0} . T_{s}$ is the sweep time, $T$ is the sweep period, $q$ is the pulse period, $T_{p}$ is the pulse width and $\Delta f$ is the bandwidth of baseband filter.

Let $\alpha=m \alpha_{A}=n \alpha_{B}, f_{0}=m f_{A 0}-n f_{B 0}, f_{Z D}=\alpha \tau_{Z D}, f_{T}=\alpha T$, then equation (3) can be written as

$$
f_{B c p}(t)= \begin{cases}f_{Z D}+f_{0}-\alpha \tau_{0} \pm l f_{q} & 0 \leq t-k T \leq T_{s}-\tau_{0}+\tau_{Z D} \\ f_{Z D}+f_{0}-\alpha \tau_{0} \pm l f_{q}+f_{T} & T-\tau_{0}+\tau_{Z D} \leq t-k T \leq T_{s}\end{cases}
$$

Because $f_{T}>S f_{q}$, there is only one equation in (4) may meet $0 \leq f_{B c p}(t)<$ $f_{q}, p \leq S$.

Similarly, we have

$$
f_{A c p}(t)= \begin{cases}f_{Z D}-\left(f_{0}-\alpha \tau_{0} \pm l f_{q}\right) & \tau_{Z D} \leq t-k T \leq T_{s}-\tau_{0}+2 \tau_{Z D} \\ f_{Z D}-\left(f_{0}-\alpha \tau_{0} \pm l f_{q}+f_{T}\right) & T-\tau_{0}+2 \tau_{Z D} \leq t-k T \leq T_{s}+\tau_{Z D}\end{cases}
$$

From equation (4) and (5) we know that $-f_{q}+2 f_{Z D}<f_{A c p}(t) \leq 2 f_{Z D}$ when $0 \leq f_{B c p}(t)<f_{q}, p \leq S$. Because $2 f_{Z D}<f_{q}$, the IDWI received by Station A also meets $-f_{q}<f_{A c p}(t)<f_{q}, p \leq S$.

\subsection{Conditions and probability of the generation of IDWI}

To get a large INR of frequency component of IDWI at baseband, the working parameters of IDWI shall meet following conditions: 


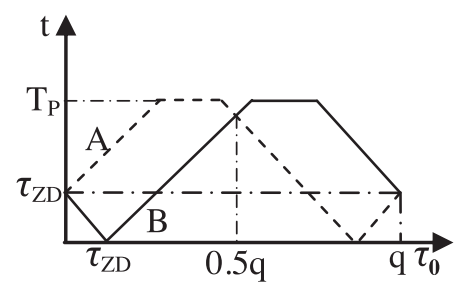

Fig. 2. Time Utilization of IDWI.

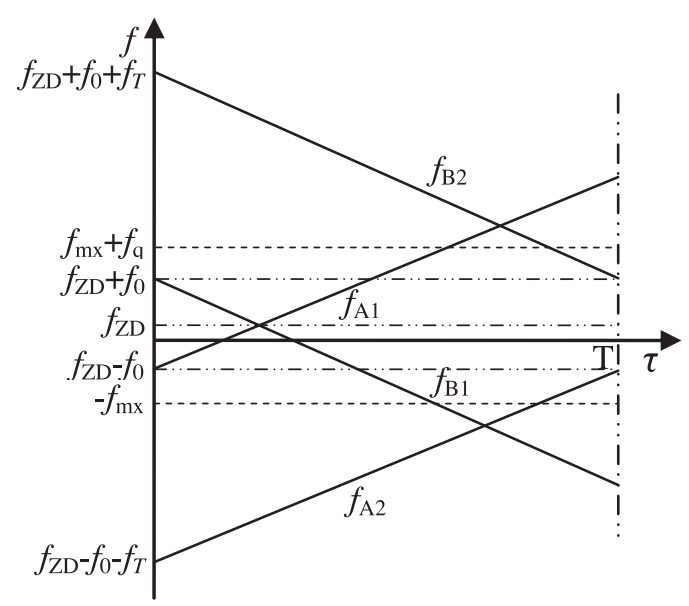

Fig. 3. Graph of function.

1) $m \alpha_{A}=n \alpha_{B}$, where $m$ and $n$ are the smallest natural numbers that meet the equation;

2) The time utilization rate of IDWI is shown in Fig. 2, and $\tau_{0}$ shall meet $\tau_{0}-r q \neq \tau_{Z D}, \tau_{0}-r q \neq q-\tau_{Z D}$ where $r=0,1,2,3, \cdots$ and $0<\tau_{0}<T$;

3) Let $f_{m x}=(S-m-n) f_{q}$ and $\tau_{0}$ be an independent variable. Set

$$
\begin{aligned}
& f_{B 1}\left(\tau_{0}\right)=f_{Z D}+f_{0}-\alpha \tau_{0} \\
& f_{B 2}\left(\tau_{0}\right)=f_{B 1}\left(\tau_{0}\right)+f_{T} \\
& f_{A 1}\left(\tau_{0}\right)=f_{Z D}-\left(f_{0}-\alpha \tau_{0}\right) \\
& f_{A 2}\left(\tau_{0}\right)=f_{A 1}\left(\tau_{0}\right)-f_{T}
\end{aligned}
$$

The graph of function is shown in Fig. 3. We can conclude from the graph that in order to meet $-f_{q}<f_{A c p}, f_{B c p}<f_{q}, p \leq S$ without affecting the probability for the generation of IDWI, two conditions shall be satisfied as follow:

Or

$$
\begin{aligned}
& f_{Z D}+f_{0}+f_{T}>f_{m x}+f_{q} \\
& f_{Z D}+f_{0}-f_{T}<2 f_{Z D}-f_{m x}-f_{q} \\
& \left|f_{0}\right|<f_{Z D}+f_{T}-f_{m x}-f_{q}
\end{aligned}
$$

The probability for the generation of IDWI is $\left(2 f_{m x}+f\right) / f_{T}$.

\subsection{Characteristics of IDWI}

IDWI has following characteristics under ideal condition: 
a) There are two IDWIs in each station and the frequencies are $f_{L}$ and $f_{H}$ respectively, where $-f_{q}<f_{L} \leq 0,0 \leq f_{H}<f_{q}$, and $f_{H}-f_{L}=f_{q}$. There are two IDWIs when $\Delta f \geq f_{q}$; there is one IDWI and the other one is filtered when $f_{q} / 2 \leq \Delta f<f_{q}$ and $f_{H}<f_{q}-\Delta f$; there are two IDWIs when $f_{q}-\Delta f \leq f_{H}$; there are two IDWIs when $f_{q} / 2 \leq \Delta f<f_{q}$ and $f_{q}-\Delta f \leq f_{H}$; there is only one IDWI when $\Delta f<f_{q} / 2$;

b) IDWIs of the two stations are symmetric on $f_{Z D}$.

The operations are independent in two stations under an asynchronous condition and time synchronization is not done. The operating parameters (operating frequency and sweep bandwidth are not included) are set to be the same, but the difference of equipment can lead the sweep period $\mathrm{T}$ of two station to be inconsistent (which means $\tau_{0}$ is not a constant but a variable). The values of $f_{A c p}$ and $f_{B c p}$ aren't stable but changeable as the time changes because of this situation. The change is gradual but not sudden.

We can conclude from equation (4) and (5) that the frequencies of IDWI under asynchronous condition are

$$
\begin{aligned}
& f_{B c p}(k)=f_{Z D}+f_{v}(k) \\
& f_{A c p}(k)=f_{Z D}-f_{v}(k)
\end{aligned}
$$

Where $k=1,2,3, \cdots$ is the frame number, $f_{v}(k)=f_{0}-\alpha \tau_{0}(k) \pm l f_{q}$ or $f_{v}(k)=f_{0}-\alpha \tau_{0}(k) \pm l f_{q}+f_{T} . f_{A c p}(k)$ and $f_{B c p}(k)$ meet $-f_{q}<f_{A c p}(k)$, $f_{B c p}(k)<f_{q}, p \leq S$.

Thus, the IDWI under asynchronous condition has characteristics as follow:

c) The IDWIs of the two stations don't have a stable frequency. The frequencies change as the time changes. The IDWIs of the two stations have the same velocity and their moving directions are opposite.

d) The IDWIs of the two stations will encounter on $f_{Z D}$ at a certain time.

\section{Calibration algorithm of asynchronous IDWI}

1) Read distance echo spectra of frame $K$ of Station A;

2) Judge the relationship of $\Delta f$ and $f_{q}$;

3) Search peaks of which INR is larger than PG frame by frame in $\left(-f_{q}, f_{q}\right)$ when $\Delta f \geq f_{q}$, and the distance between two peaks is $f_{q}$, then we get $a(k)$, where $k=1,2, \cdots, K$ and $\mathrm{PG}$ is the preset threshold value of ING;

4) Search peaks of which ING is larger than PG frame by frame in $\left(-f_{q},-\Delta f\right)$ and $\left(\Delta f, f_{q}\right)$ when $f_{q} / 2 \leq \Delta f<f_{q}$, and the distance between two peaks is $f_{q}$; search peaks of which ING is larger than PG frame by frame in $[-\Delta f, \Delta f]$, then we get $a(k)$;

5) Search peaks of which ING is larger than PG frame by frame in $[-\Delta f, \Delta f]$ when $\Delta f<f_{q} / 2$, then we get $a(k)$;

6) Get $a(k)$ according to step 3$)$ then choose a series of gradual changing data $z(k)$ from $a(k)$; 
7) Get $a(k)$ according to step 4) or step 5), read $a(k)$ which records locations of peaks in the same time from Station B;

8) Compare $a(k)$ and $a^{\prime}(k)$, choose a series of gradual changing data $z(k)$ which are symmetric on $f_{Z D}$ from $a(k)$, choose a series of gradual changing data $z^{\prime}(k)$ which are symmetric on $f_{Z D}$ from $a^{\prime}(k)$, and transmit $z^{\prime}(k)$ to Station B;

9) Get $z(k)$ according to step 6) or step 8), Use $z(k)$ to construct interstation direct wave receiving data $\mathbf{Y}(k)=\left[Y_{1}(z(k)), Y_{2}(z(k)), \cdots, Y_{N}(z(k))\right]^{T}$ where $Y_{i}(z(k))$ is IDWI of frame $\mathrm{k}$ of the ith channel and $\mathrm{N}$ is the number of channels;

10) Estimate covariance matrix $\widehat{\mathbf{R}}=\frac{1}{K} \sum_{k=1}^{K} \mathbf{Y}(k) \mathbf{Y}^{H}(k)$;

11) Get eigenvector $\mathbf{e}_{1}$ by decomposition;

12) Find array gain-phase errors $\boldsymbol{\Gamma}$ from $\boldsymbol{\Gamma} \mathbf{a}\left(\theta_{S}\right)=u \mathbf{e}_{1}$, where $\mathbf{a}\left(\theta_{S}\right)$ is the steering vector of direct wave and $u$ is an unknown constant complex number.

\section{Experiment of calibration of asynchronous IDWI}

We implemented experiment of calibration of IDWI at shallow water of North of Jiangsu in China on July 24, 2011. We set Taiyangdao Station and

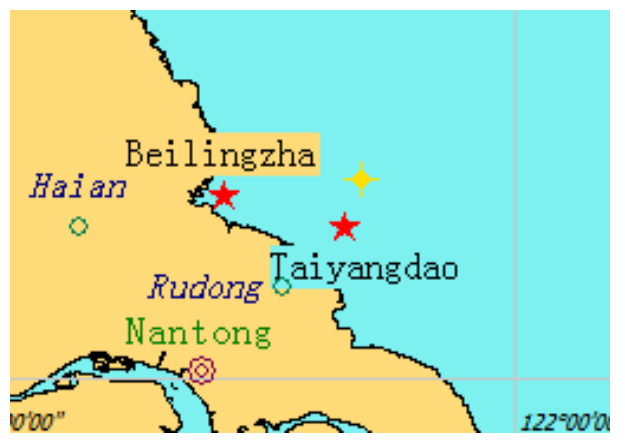

Fig. 4. Locations of Experimental Stations.

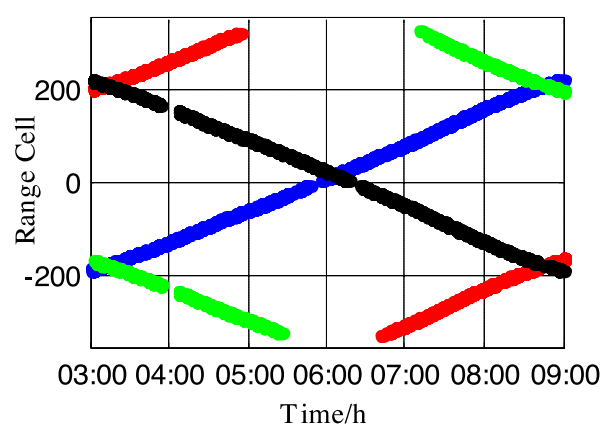

Fig. 5. Range cell information of IDWI of the two stations, Red and Blue are the first and second IDWI of Taiyangdao Station, respectively. Green and Black are the first and second IDWI of Beilingzha Station, respectively. 
Beilingzha Station as experimental stations of which locations are shown in Fig. 4. The working parameters of radars at the two stations are the same during experiment and specific parameters are as follow: the initial frequency
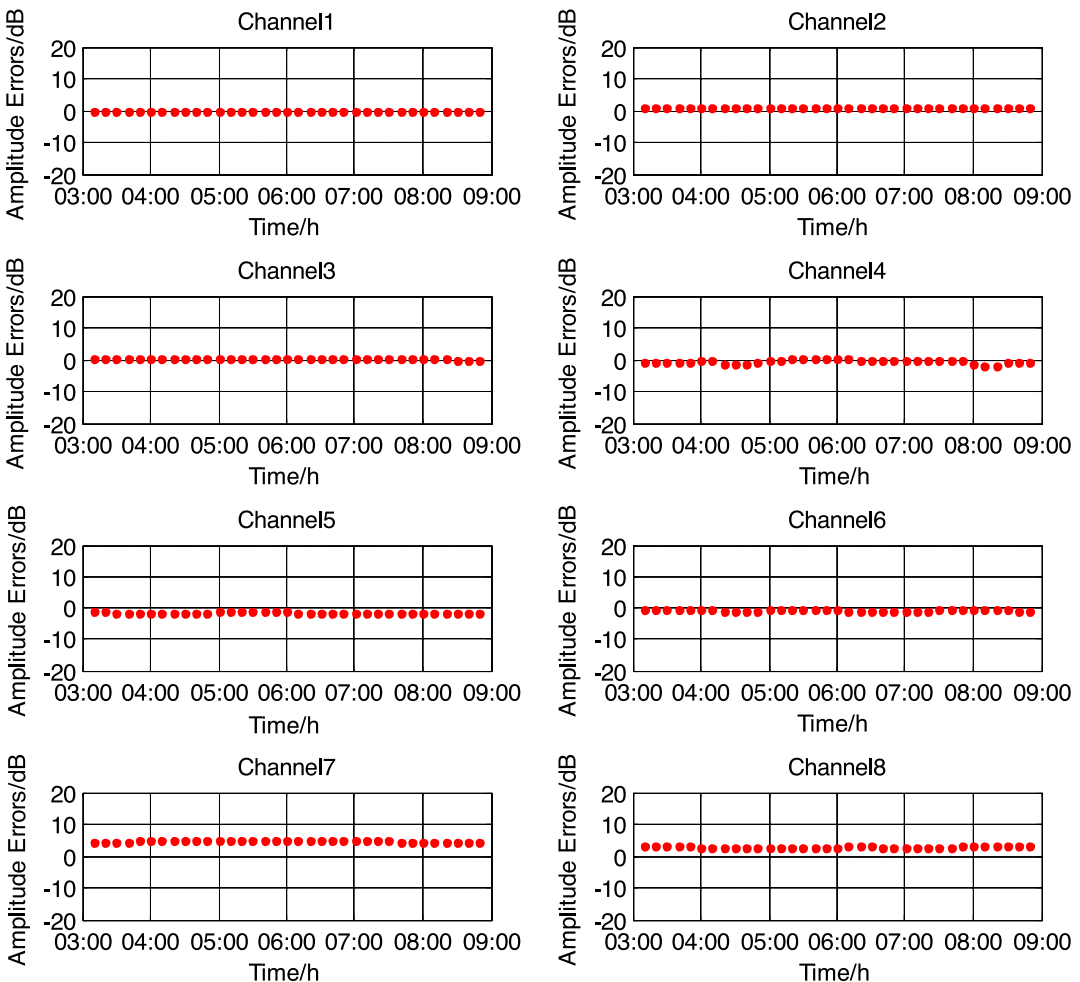

Fig. 6. Calibration Value Taiyangdao Station.

of Amplitude Errors of
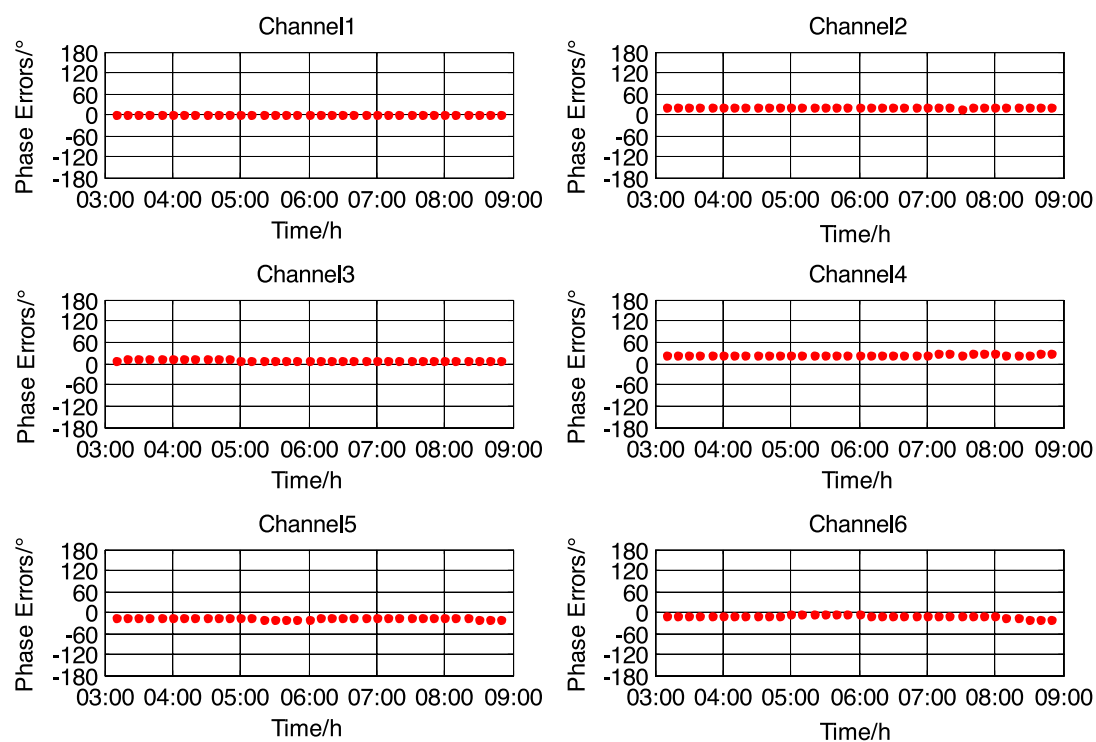
Time/h
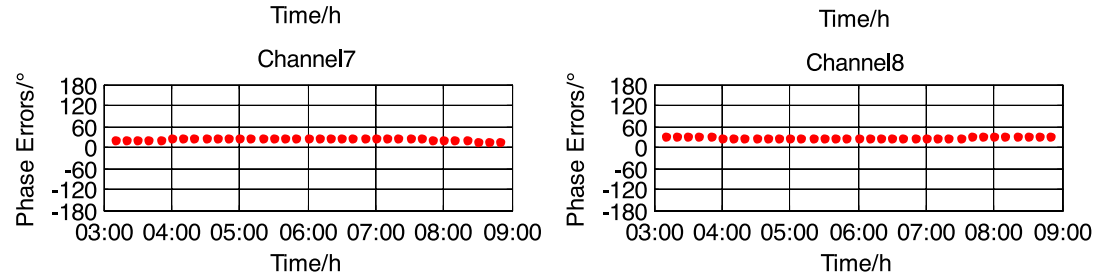

(c) IEICE 2012

DOI: $10.1587 /$ elex.9.450 Received January 11, 2012 Accepted February 13, 2012 Published March 25, 2012 
is $11.22 \mathrm{MHz}$, sweep time is $0.6144 \mathrm{~s}$, sweep period is $0.6528 \mathrm{~s}$, sweep bandwidth is $120 \mathrm{KHz}$, ange resolution is $1.25 \mathrm{~km}$, pulse period is $1.6 \mathrm{~ms}$, pulse width is $0.8 \mathrm{~ms}$, bandwidth of baseband filter is $500 \mathrm{~Hz}$, number of sampling points in sweep time is 1024 and the distance between the two station is $41.73 \mathrm{~km}$.

Results of the experiment are shown in Fig. $5 \sim 7$. We can see that the IDWI of the two stations meet its characteristics from Fig. 5. We can see the calibration value of range errors of Taiyangdao Station is stable from Fig. 6 and the standard deviation of amplitude errors is about $0.2 \mathrm{~dB}$. The biggest standard deviation of amplitude errors, which is on Channel 4, reaches to $0.6 \mathrm{~dB}$. We can see the calibration value of phase errors of Taiyangdao Station is stable from Fig. 7 and the standard deviation of phase errors is about $1.5^{\circ}$. The biggest standard deviations of phase errors, which are on Channel 6 and Channel 7 , reach to $4^{\circ}$.

\section{Conclusion}

The method proposed in this paper can implement active calibration without auxiliary source and perform outstanding precision and stability. The analysis of experimental data demonstrates that this method is effective and feasible. Using the calibration value obtained from the calibration method based on IDWI to implement the amplitude compensation and phase compensation of the sea echo data provides strong support of inversion of ocean surface dynamics parameters of high frequency radar system.

\section{Acknowledgments}

This research was supported by the Eleventh-Five-Year Chinese HighTechnology 863 Plan Project under grant 2009AA09A301, the National Scientific Fund Committee (NSFC) under grant 60571065 and the Fundamental Research Fund for the Central Universities. 